\title{
Growth and characterization of barium copper oxalate single crystals in gels
}

\author{
KASTHURI V BANGERA and P MOHAN RAO* \\ Department of Physics, K R E College, Surathkal, Srinivasnagar 574 157, India \\ *Department of Physics, Mangalore University, Mangalagangothri 574 199, India \\ MS received 10 November 1991; revised 28 January 1992
}

\begin{abstract}
Barium copper oxalate was grown in silica hydrogel at ambient temperature. The effect of various parameters like gel $\mathrm{pH}$, gel density, gel aging and concentrations of reactants on the growth of these crystals was studied. The crystals grown were characterized by chemical analysis, X-ray powder diffractometry, infrared spectroscopy, thermogravimetric and differential thermal analysis. The results of these observations are described and discussed.
\end{abstract}

Keywords. Barium copper oxalates; silica hydrogel; gels.

\section{Introduction}

In recent years crystal growth in gel medium has attracted the attention of many investigators (Venkateswara Rao 1985; Dharma Prakash and Mohan Rao 1986; Kotru et al 1986; Narayana Kalkura and Deva Narayan 1987). The principle relies on the slow migration of crystal constituents (ions) through silica hydrogel so that a very slow reaction occurs with the formation of a sparingly soluble compound. When the concentration of this compound exceeds the solubility limits, crystals will be formed, the main function of the gel being to control the flow of reacting ions.

Mixed crystal growth has scarcely been studied by employing the gel technique (Joshi et al 1980; Dharma Prakash and Mohan Rao 1986) and the field is in an early stage of development with many opportunities to create new species. The purpose of the present paper is to report the growth of single crystals of barium copper oxalate in silica hydrogel at ambient temperature.

\section{Experimental}

The growth process involved diffusion of barium chloride and copper chloride solutions into a gel in which oxalic acid was impregnated before hand. Crystal growth experiments were carried out in corning glass tubes (length $200 \mathrm{~mm}$, inner diameter $25 \mathrm{~mm}$ ). The chemicals used were commercial sodium metasilicate (water glass) solution, oxalic acid (AnalaR), barium chloride (AnalaR) and copper chloride (AnalaR). The gel was set by mixing sodium meta silicate solution of specific gravity 1.04 with aqueous $1.0 \mathrm{M}$ oxalic acid in proper ratio such that the $\mathrm{pH}$ of the resulting solution was around $3 \cdot 1$. After the gel was set a mixture of barium chloride and copper chloride solution of varying strengths $(0 \cdot 25-1 \mathrm{M})$ was slowly poured above the set gel down the walls of different test tubes. The crystals grown were characterized

\footnotetext{
*For correspondence
} 
by chemical analysis, X-ray powder diffraction, infrared spectroscopy, thermogravimetric and differential thermal analyses.

\section{Observations and discussion}

In terms of clarity of gel mixture and separation between the crystals, the combination of $\mathrm{pH} 2.9$ to 3.2 gel mixture and $0.5 \mathrm{M}$ supernatant feed solution (barium chloride and copper chloride) gave the best results. The specific gravity of the sodium meta silicate solution used was 1.04 . The gelling time was about one week. Nucleation was observed within a day after adding the chloride solutions to the gel set with $1.0 \mathrm{M}$ oxalic acid just below the gel interface. It was observed that barium copper oxalate crystals are grown in the form of transparent blue crystals of cubic shape near the interface. As the mixed chemical solution diffuses deep into the gel, transparent colourless crystals of different morphology were found to grow. These transparent blue and colourless crystals are henceforth referred to as types I and II crystals respectively. A typical experimental tube where types I and II crystals were grown is shown in figure 1 . The growth was completed in about three weeks. The average size of the type I crystals was about $5 \times 3 \mathrm{~mm}$ and that of type II crystals was about $10 \times 3 \mathrm{~mm}$. Some of the crystals grown are shown in figures 2 and 3 .

\section{$3.1 p H$}

It is found that the initial $\mathrm{pH}$ values of 2.9-3.1 give the optimum condition for the growth of barium copper oxalate crystals. When the $\mathrm{pH}$ was increased above $3 \cdot 4$, irregularly shaped and opaque dendritic crystals were obtained along with the formation of Leisgang rings. On the other hand it was observed that only few blue crystals were formed below $\mathrm{pH} 2 \cdot 6$.

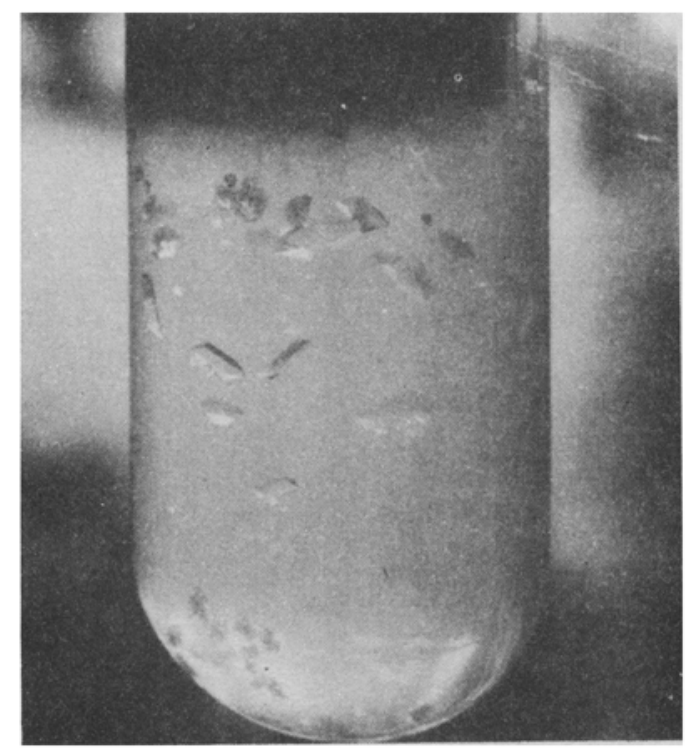

Figure 1. A typical growth tube $(\times 3)$. 


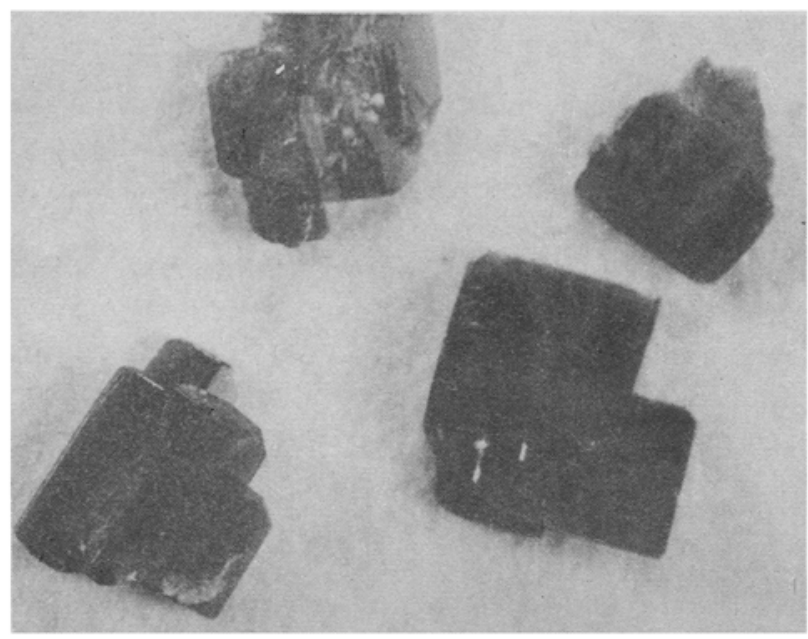

Figure 2. Transparent blue crystals of $\mathrm{Ba}_{x} \mathrm{Cu}_{1-x} \mathrm{C}_{2} \mathrm{O}_{4} 4 \mathrm{H}_{2} \mathrm{O}$ (type 1) (×5.5).

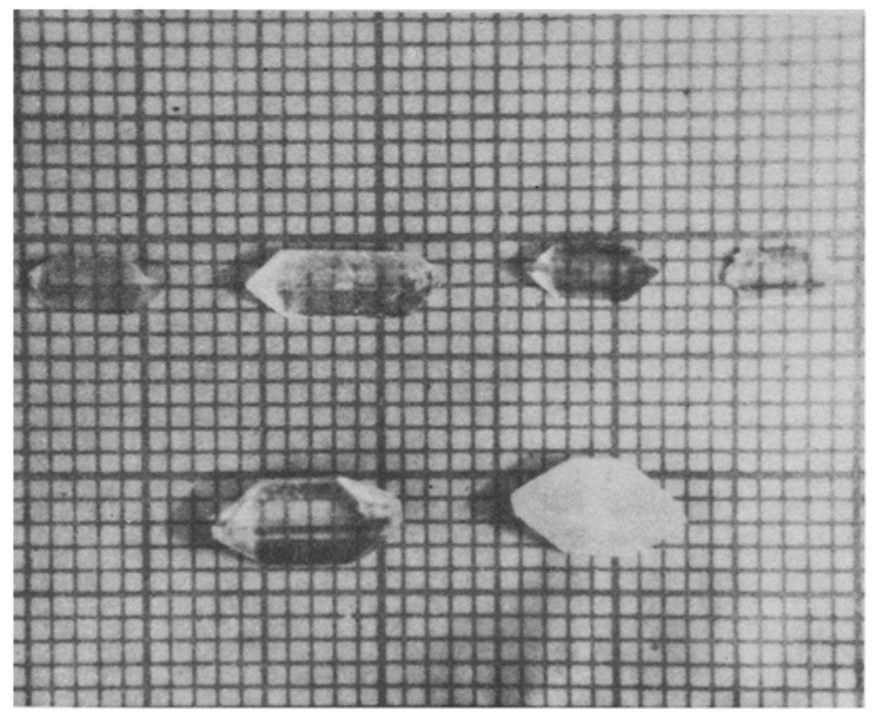

Figure 3. Well-developed colourless crystals of $\mathrm{Ba}_{x} \mathrm{Cu}_{1-x} \mathrm{C}_{2} \mathrm{O}_{4} 4 \mathrm{H}_{2} \mathrm{O}$ (type II).

\subsection{Gel density}

In general, very dense gels produce poor crystals. On the other hand, gels of insufficient density take a longer time to form and are mechanically unstable. The present observations agree with this general rule. Generally, for growing crystals, gel density from 1.03 to 1.06 can be used. It is observed that nucleation density decreases as the gel density increases. A greater gel density implies smaller pore size and poor communication among the pores thus decreasing the nucleation density (Helberstadt et al 1969). 


\subsection{Gel aging}

Aging of gels decreases the pore size and hence decreases the diffusion and nucleation density. The longer a gel is set at room temperature, the greater the amount of water which evaporates from the gel. The effect of evaporation of water should be considered before and after the formation of gel framework. Before the gel is set, the evaporation of water causes an increase in gel density which in turn decreases the diffusivity of $\mathrm{Ba}^{2+}$ and $\mathrm{Cu}^{2+}$ ions in the gel, thereby decreasing the number of nucleation sites. After the gel is set, evaporation of water causes not only the lack of ionic carriers in the channel of gel framework, but also discontinuities in the channel due to the shrinkage of the gel. Both these effects would adversely affect the diffusion of $\mathrm{Ba}^{2+}$ and $\mathrm{Cu}^{2+}$ and hence the number of nucleation sites.

\subsection{Concentration of reactants}

It is seen that as the concentration of the feed solutions increases, the nucleation density also increases. This may be due to the enhanced availability of $\mathrm{Ba}^{2+}$ and $\mathrm{Cu}^{2+}$ ions. To grow good crystals, the concentration of feed solution suitable is $0.5 \mathrm{M}$. Similarly the concentration of oxalic acid suitable for the growth of good quality crystals is $1.0 \dot{M}$.

\subsection{Chemical analysis}

Both varieties of crystals, viz. types I and II crystals were subjected to chemical analysis. The results are shown in table 1 .

\subsection{X-ray powder diffractometric studies}

$\mathrm{X}$-ray powder diffraction pattern of these crystals was recorded with X-ray diffractometer (JEOL, JDX OP) using $\mathrm{FeK}_{\alpha}$ radiation. The samples were scanned over a $2 \theta$ range of $10^{\circ}$ to $100^{\circ}$ at $2^{\circ} / \mathrm{min}$. The observed $d$ values with relative intensities for the two varieties of crystals are given in tables 2 and 3.

\subsection{Infrared absorption spectra}

Infrared absorption spectra of both these gel grown crystals were recorded on a spectrophotometer (Shimadzu IR-408) using $\mathrm{KBr}$ pellet technique. The infrared spectra for the two varieties of crystals are shown in figures 4 and 5 .

Table 1. Chemical composition of types I and II crystals.

\begin{tabular}{lll}
\hline Sample & $\mathrm{Ba}^{2+}$ & $\mathrm{Cu}^{2+}$ \\
\hline Type I (bluish) crystal & $28 \cdot 24 \%$ & $16 \cdot 38 \%$ \\
Type II (colourless) crystal & $47 \cdot 77 \%$ & $12 \mathrm{ppm}$ \\
\hline
\end{tabular}


Table 2. Observed $d$ values with relative intensities for type I barium copper oxalate crystal.

\begin{tabular}{llcl}
\hline$d\left(10^{-8} \mathrm{~cm}\right)$ & Intensity & $d\left(10^{-8} \mathrm{~cm}\right)$ & Intensity \\
\hline 6.277 & S & 2.967 & S \\
5.899 & S & 2.684 & W \\
5.577 & W & 2.405 & W \\
5.340 & VS & 2.347 & W \\
4.519 & VW & 2.240 & VW \\
4.249 & S & 2.126 & W \\
3.969 & W & 1.737 & VW \\
3.773 & VW & 1.694 & W \\
3.471 & S & 1.527 & S \\
3.185 & VVW & 1.291 & VS \\
\hline
\end{tabular}

VW, very weak; W, weak; $S$, strong; VS, very strong.

Table 3. Observed $d$ values with relative intensity for type II barium copper oxalate crystal.

\begin{tabular}{lccc}
\hline$d\left(10^{-8} \mathrm{~cm}\right)$ & Intensity & $d\left(10^{-8} \mathrm{~cm}\right)$ & Intensity \\
\hline 6.689 & VW & 2.960 & W \\
6.295 & VS & 2.845 & VS \\
5.930 & VW & 2.441 & S \\
5.316 & S & 2.315 & VS \\
4.492 & S & 2.184 & VS \\
4.226 & W & 1.967 & W \\
3.754 & VS & 1.890 & S \\
3.313 & VS & 1.772 & W \\
3.177 & VS & 1.524 & VS
\end{tabular}

VW, very weak; S, strong; W, weak; VS, very strong.

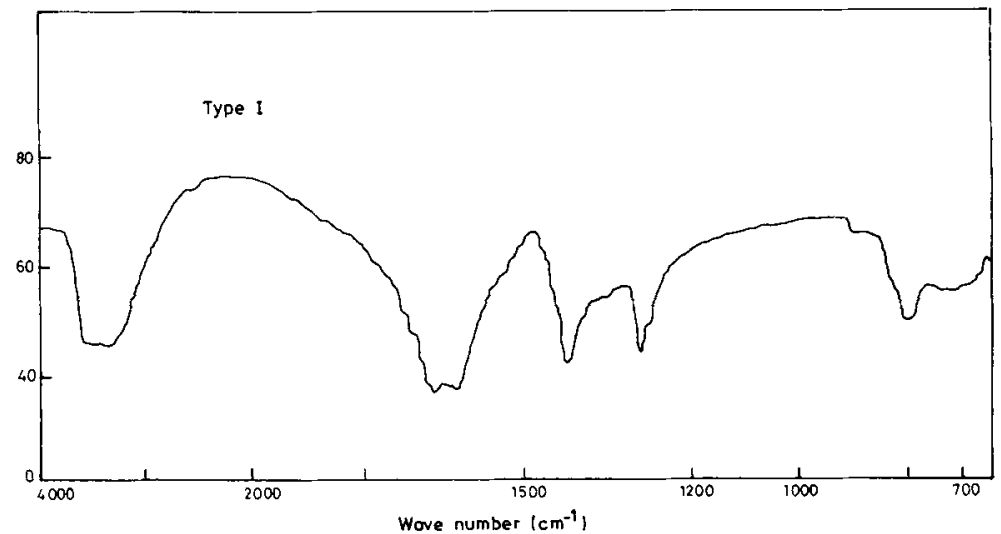

Figure 4. Infrared spectra of $\mathrm{Ba}_{x} \mathrm{Cu}_{1-x} \mathrm{C}_{2} \mathrm{O}_{4} 4 \mathrm{H}_{2} \mathrm{O}$ (type I). 


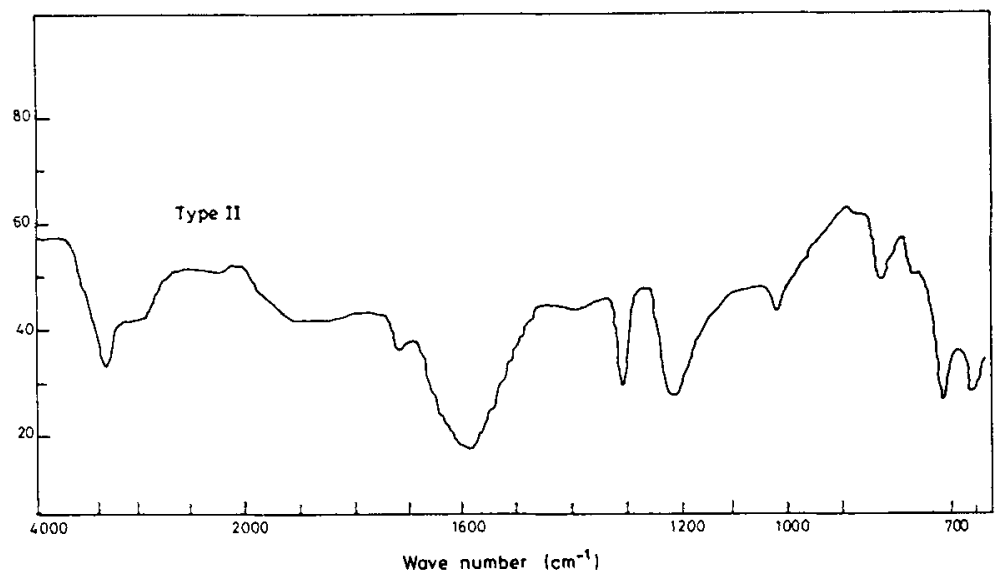

Figure 5. Infrared spectra of $\mathrm{Ba}_{x} \mathrm{Cu}_{1-x} \mathrm{C}_{2} \mathrm{O}_{4} 2 \mathrm{H}_{2} \mathrm{O}$ (type II).

Table 4. Assignment of IR band frequencies $\left(\mathrm{cm}^{-1}\right)$.

\begin{tabular}{|c|c|c|}
\hline $\begin{array}{l}\mathrm{BaCu} \mathrm{C}_{2} \mathrm{O}_{4} 4 \mathrm{H}_{2} \mathrm{O} \\
\text { (type I crystal) }\end{array}$ & $\begin{array}{c}\mathrm{BaCu} \mathrm{C}_{2} \mathrm{O}_{4} 2 \mathrm{H}_{2} \mathrm{O} \\
\text { (type II crystal) }\end{array}$ & Assignment \\
\hline 3400 & 3350 & $(\mathrm{OH})$ stretch \\
\hline 1650 & 1600 & (HOH) bend \\
\hline 1425 & - & \\
\hline \multirow[t]{4}{*}{1295} & 1310 & $(\mathrm{C}-\mathrm{O})$ \\
\hline & & asymmetric stretch \\
\hline & 1215 & \\
\hline & 1020 & \\
\hline \multirow[t]{3}{*}{810} & 840 & $(\mathrm{M}-\mathrm{O})$ \\
\hline & $715)$ & 10 \\
\hline & $645\}$ & $(0-C-0)$ \\
\hline
\end{tabular}

Simple oxalate salts consist of a metal cation and the oxalate anion. Therefore, the infrared spectra of these crystals are considered to arise from the oxalate ion and from the crystallization water present in the crystal. IR studies on various oxalates have been carried out by several investigators (Freeberg et al 1967; Shippy 1980a). The observed infrared bands and their assignments are shown in table 4.

In agreement with Shippy (1980b), the bands above $3000 \mathrm{~cm}^{-1}$ are assigned to $\mathrm{OH}$ stretching vibrations of water molecules. The $\mathrm{H}-\mathrm{O}-\mathrm{H}$ bending mode has been assigned to the IR band at about $1600 \mathrm{~cm}^{-1}$. These two bands for the two crystals are almost similar, suggesting that the bonding of water molecules in the two crystals is similar and the $\mathrm{O}-\mathrm{H} \ldots \mathrm{O}$ distances may be almost the same. The bands around $1300 \mathrm{~cm}^{-1}$ correspond to the asymmetric stretching mode of $\mathrm{C}-\mathrm{O}$ bond. A slight difference in the frequencies of this band in the crystals indicates that the $\mathrm{Ba}-\mathrm{O}$ and $\mathrm{Cu}-\mathrm{O}$ bond lengths in the two structures are not similar. The observed IR bands around 715 and $645 \mathrm{~cm}^{-1}$ are assigned to various fundamental modes of vibrations of $\mathrm{O}-\mathrm{C}-\mathrm{O}$ bond.

The IR band observed at $840 \mathrm{~cm}^{-1}$ in type II crystals and at $810 \mathrm{~cm}^{-1}$ in type I crystals has been assigned to $\mathrm{M}-\mathrm{O}$ bond (Packter and Omon 1984) where $\mathrm{M}=\mathrm{Ba}+\mathrm{Cu}$. 
The infrared spectral studies described above confirm the general crystal structure for simple metal oxalates (Fujita 1957) and represented schematically as:<smiles>[M]OC(=O)OC=O</smiles>

where $\mathrm{M}=\mathrm{Cu}+\mathrm{Ba}$.

\subsection{Thermogravimetric and differential thermal analysis}

Differential thermal analysis (DTA) and thermogravimetric analysis (TGA) of these gel-grown barium copper oxalate crystals were carried out simultaneously by employing an automatic recording apparatus (Shimadzu DTA-30) at a heating rate of $10^{\circ} \mathrm{C} \mathrm{min}^{-1}$. The typical TGA and DTA curves are shown in figures 6 and 7 .

The number of water molecules present and the purity of the crystals were determined from thermogravimetric analysis. Taking either the weight percentage of the end product or the initial weight as standard, the course of decomposition was analysed comparing it with the molecular weight. It is observed that types I and II crystals of barium copper oxalate contain 4 and 2 molecules of water respectively.

TGA and DTA data of various oxalates (Dollimore et al 1963, 1970) showed that most oxalates decompose in nitrogen producing the respective metals as final products in a single reaction. Since the present decomposition study was carried out in air, the end product would certainly not reach the stage of metals barium or copper.

The DTA curve of type II barium copper oxalate (figure 7) shows endothermic peak at about $170^{\circ} \mathrm{C}$, two sharp exothermic peaks at around $235^{\circ} \mathrm{C}$ and $435^{\circ} \mathrm{C}$ and a small endothermic peak at $780^{\circ} \mathrm{C}$.

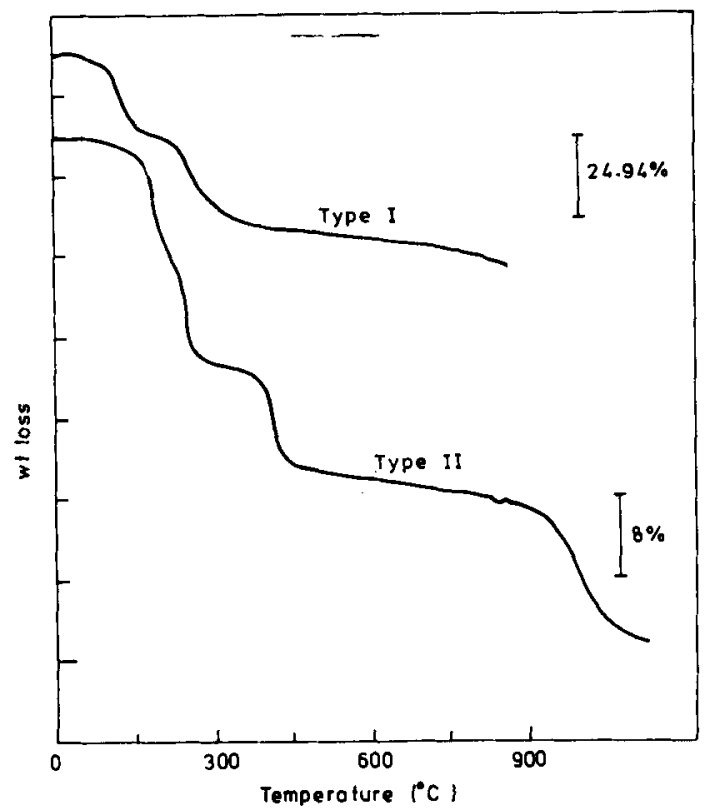

Figure 6. TGA plot of barium copper oxalate. 


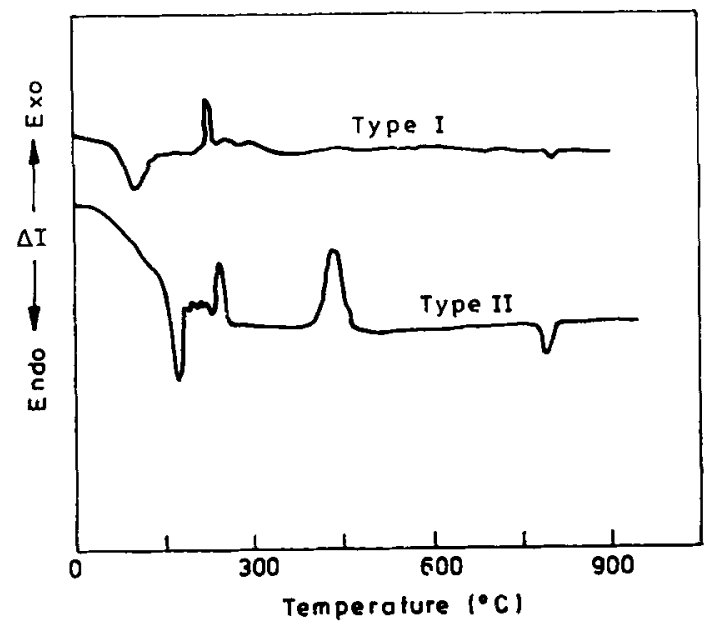

Figure 7. DTA plot of barium copper oxalate.

The endothermic peak at $170^{\circ} \mathrm{C}$ must correspond to the liberation of water molecules from the dehydrated $\mathrm{Ba}_{x} \mathrm{Cu}_{1-x} \mathrm{C}_{2} \mathrm{O}_{4}$ which undergoes decomposition to form $\mathrm{BaCO}_{3}$ and $\mathrm{CuO}$. The exothermic peak at around $235^{\circ} \mathrm{C}$ corresponds to the formation of $\mathrm{CuO}$ and the peak around $435^{\circ} \mathrm{C}$ indicates the formation of $\mathrm{BaCO}_{3}$. The endothermic peak at $780^{\circ} \mathrm{C}$ is associated with the decomposition of $\mathrm{BaCO}_{3}$ to $\mathrm{BaO}$.

Similarly, the DTA curve of type I crystals (figure 7) shows endothermic peak at about $90^{\circ} \mathrm{C}$ and a exothermic peak at $220^{\circ} \mathrm{C}$ and a small endothermic peak at about $770^{\circ} \mathrm{C}$.

The endothermic peak at $90^{\circ} \mathrm{C}$ in the DTA curve (figure 7) corresponds to dehydration of the mixed oxalate crystals. The exothermic peak around $220^{\circ} \mathrm{C}$ must be due to the transformation of copper oxalate into copper oxide. The small endothermic peak at $770^{\circ} \mathrm{C}$ corresponds to the decomposition of barium oxalate into barium oxide. It may be noted here that as the copper concentration in the mixed crystal increases (i.e. I type of crystals), the DTA peak corresponding to the decomposition of barium oxalate into barium carbonate is absent. This may be attributed to the fact that, the initial decomposition reaction to copper oxide is so exothermic that the subsequent decomposition of unreacted barium oxalate is accelerated.

\section{Conclusion}

Barium copper oxalate mixed crystals in silica hydrogel have been grown successfully at ambient temperature. It is found that well-developed single crystals are obtained at $0.5 \mathrm{M}$ concentrations of feed solution in the $\mathrm{pH}$ range 2.9 to 3.1 of the gel.

\section{Acknowledgement}

We thank Prof. G K Shivakumar, Head, Department of Physics, K R E College, Surathkal for encouragement and laboratory facilities. The authors are also thankful 
to the Metallurgical Engineering Department and Chemistry Department for assistance in analytical work.

\section{References}

Dharma Prakash S M and Mohan Rao P 1986 J. Mater. Sci. Lett. 5769

Dollimore D, Griffiths D L and Nicholson D 1963 J. Chem. Soc. 2617

Dollimore D and Griffiths D L 1970 J. Therm. Anal. 2229

Freeberg F E, Hartman K O, Hisatune I C and Schempf J M 1967 J. Phys. Chem. 71397

Fujita M 1957 J. Phys. Chem. 611014

Helberstadt E S, Henisch H K, Nickl J and White E W 1969 J. Colloid Interface Sci. 29469

Joshi M S, Mohan Rao P and Antony A V 1980 Bull. Mater. Sci. 2127

Kotru P N, Raina K K and Koul M L 1986 J. Mater. Sci. 21393

Narayana Kalkura S and Deva Narayanan S 1987 J. Cryst. Growth 83446

Packter A and Omon A 1984 Crystal Res. Technol. 19467

Shippy T A 1980a J. Mol. Struct. 63157

Shippy T A 1980b J. Mol. Struct. 67223

Venkateswara Rao A 1985 Bull. Mater. Sci. 783 